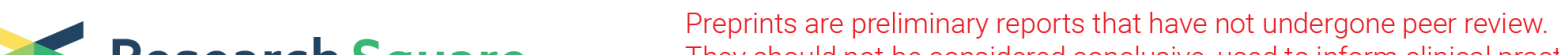 Research Square They should not be considered conclusive, used to inform clinical practice, or referenced by the media as validated information.
}

\section{Association of Preadmission Metformin Exposure and Outcomes in Acute Kidney Injury Patients with Type 2 Diabetes in Intensive Care Unit: a Cohort Study}

\section{Qilin Yang}

The Second Affiliated Hospital of Guangzhou Medical University

Jiezhao Zheng

The Second Affiliated Hospital of Guangzhou Medical University

\section{Deliang Wen}

The Second Affiliated Hospital of Guangzhou Medical University

\section{Xiaohua Chen}

The Second Affiliated Hospital of Guangzhou Medical University

\section{Weiyan Chen}

The Second Affiliated Hospital of Guangzhou Medical University

\section{Weixiao Chen}

The Second Affiliated Hospital of Guangzhou Medical University

\section{Xuming Xiong}

The Second Affiliated Hospital of Guangzhou Medical University

\section{Zhenhui Zhang ( $\nabla$ zhzhhicu@126.com)}

The Second Affiliated Hospital of Guangzhou Medical University

\section{Research}

Keywords: Metformin, Mortality, AKI, Type 2 diabetes, NLR

Posted Date: August 20th, 2020

DOl: https://doi.org/10.21203/rs.3.rs-54095/v1

License: (c) (i) This work is licensed under a Creative Commons Attribution 4.0 International License. Read Full License 


\section{Abstract}

Background: Acute kidney injury (AKI) occurred in more than half of ICU patients. The effective prevention and therapies strategy of AKI remains limited. This study is to assess AKI mortality among diabetes patients with or without preadmission prescriptions for metformin.

Methods: We included AKI patients with type 2 diabetes in Medical Information Mart for Intensive Care (MIMIC)-III database. 30-day mortality, neutrophil-to-lymphocyte ratio, and length of stay (LOS) in the hospital were compared between those with and without preoperative prescriptions. The statistical approaches included multivariate regression, propensity score analysis and an inverse probability-weighting model to ensure the robustness of our findings.

Results: In total, 4328 AKI patients with type 2 diabetes (998 in preadmission metformin usage group and 3330 in no preadmission metformin usage) were included in the analysis. The overall 30-day mortality was $14.2 \%$ (613/4328). There was $15.7 \%$ (523/3330) and 9.0\% (90/998) of 30-day mortality in no preadmission metformin usage and preadmission metformin usage group, respectively. In the main analysis, preadmission metformin usage was associated with a $37 \%$ lower of 30 -day mortality $(\mathrm{HR}=0.63,95 \% \mathrm{Cl}: 0.50-0.80, \mathrm{p}<0.0001)$ in inverse probability-weighting model.

Conclusions: This cohort study suggested that preadmission metformin usage may be associated with reduced risk-adjusted mortality in AKI patients with type 2 diabetes. Further randomized controlled trials are needed.

\section{Background}

Acute kidney injury (AKI) is now recognized as a major public health problem affecting millions of people worldwide and leading to decreased survival, increased progression of underlying chronic kidney disease (CKD), and sometimes to new onset of CKD[1]. The effective prevention and therapies strategy remains limited[2].

Metformin, the most common and first-line biguanide antihyperglycemic agent, has anti-inflammatory properties and is associated with lower all-cause mortality compared with other hypoglycemics $[3,4]$. Several studies demonstrated that preadmission metformin usage was associated with reduced mortality among kidney disease patients[5, 6]. Bell reported preadmission metformin was associated with a lower rate of 28-day mortality in AKI patients[7]. A recent multinational cross-sectional study demonstrated $57.3 \%$ intensive care unit (ICU) patients suffered AKI [8]. However, in critical care patients, the evidence of preadmission metformin decrease a risk of AKI mortality was still absented. Therefore we conducted a retrospective cohort study to determine the association of preadmission metformin and mortality in AKI patients with diabetes.

\section{Methods}


We enrolled a cohort of AKI patients with type 2 diabetes who were exposed or not to metformin prior to admission in the database of Medical Information Mart for Intensive Care (MIMIC)-III (version 1.4). MIMIC-III is a real-world and publicly available clinical database contained more than 60,000 intensive care unit (ICU) admissions in Beth Israel Deaconess Medical Center between 2001 and 2012[9]. We were approved to use the database. All reporting followed the Strengthening the Reporting of Observational Studies in Epidemiology (STROBE) guidelines[10].

\section{Study population}

AKI patients with type 2 diabetes were eligible in our study. AKI was defined according to the Kidney Disease: Improving Global Outcomes (KDIGO) criteria on admission were considered eligible for study inclusion. KDIGO criteria are as follows[11]: increase in serum creatinine (SCr) to $\geq 1.5$ times baseline must have occurred within the prior 7 days; or $\geq 0.3 \mathrm{mg} / \mathrm{dl}$ increase in $\mathrm{SCr}$ occurred within $48 \mathrm{~h}$; or urine volume $<0.5 \mathrm{ml} / \mathrm{kg} / \mathrm{h}$ for $6 \mathrm{~h}$ or more. The minimum of the SCr values available within the 7 days before admission was used as the baseline SCr $[12,13]$. When the preadmission SCr was not available, the first $\mathrm{SCr}$ measured at admission was used as the baseline $\mathrm{SCr}[12,14]$. The diagnosis of type 2 diabetes was based on International Classification of Disease, Ninth Revision (ICD-9). We only included adult patients (age $>16$ years). For patients admitted to the ICU more than once, only the first ICU stay was considered.

\section{Metformin Exposure}

Preadmission metformin exposure was defined as a record of using metformin in "Medications on admission" in MIMIC-III.

\section{Covariates}

We included the following variables: demographic characteristics, marital status, insurance, and service unit, heart rate, mean arterial pressure (MAP), respiratory rate, SPO2, white blood cell (WBC) count, hemoglobin, platelet, creatinine, lactate, glucose, SOFA score, simplified acute physiology score (SAPS) II score, ventilator use, vasopressor use, renal replace treatment (RRT) use, and comorbidity disease included cardiovascular disease, liver disease, malignancy, neurological disease, chronic pulmonary disease, hypertension. Vasopressor included norepinephrine, epinephrine, phenylephrine, vasopressin, dopamine, dobutamine, and Isuprel. We also included marital status and insurance. These variables included those representing the health habits of patients who received preadmission metformin that may capture a healthy user effect[15].

\section{Outcomes}


The primary outcome was 30-day mortality. The secondary outcomes were neutrophil-to-lymphocyte ratio (NLR), and length of stay (LOS) in the hospital.

\section{Statistical analysis}

A descriptive analysis was performed for all participants. Categorical variables were expressed as numbers and percentages (\%). Continuous variables were expressed as mean and standard deviation (SD) when normally distributed or median and interquartile range (IQR) when skewed. The chi-square tests (categorical variables) and One-Way ANOVA (normal distribution), Kruskal-Wallis (skewed distribution) test were used for comparison of categorical, normally, nonnormally distributed continuous variables, respectively.

To minimize the potential bias of treatment allocation and confounding, we generated a propensity score to estimate by logistic regression the likelihood that patients had preadmission metformin exposure[16]. A 1:1 nearest neighbor matching algorithm was applied using a caliper width of 0.01 . The following variables were selected to generate the propensity score: age, sex, ethnicity, marital status, insurance, admission type, service unit, heart rate, MAP, respiratory rate, SPO2, WBC, creatinine, hemoglobin, platelet, ventilator use, vasopressor use, and SAPS II score. A standardized mean difference (SMD) was used to examine the PSM degree. A threshold of less than 0.1 was considered acceptable. On the PSM cohort, we used a 2-sided t test to compare preoperative NLR and LOS in hospital. We applied the Kaplan-Meier and log-rank analyses were used for 30-day survival curves.

Using the estimated propensity scores as weights, an inverse probabilities weighting (IPW) model was used to generate a weighted cohort[17]. A Cox proportional hazards regression was then performed to adjusted propensity score. We also used a univariable Cox proportional hazards regression model with the robust variance estimator to calculate the hazard ratio (HR) for mortality.

All analyses were performed with the statistical software packages R. version 3.4.3 (R Foundation for Statistical Computing, Vienna, Austria). EmpowerStats (X\&Y Solutions, Inc., Boston, MA). The threshold of $p<0.05$ (two-sided) was considered statistically significant.

\section{Sensitivity Analyses}

To ascertain whether the results were sensitive to the matching method, we performed several additional sensitivity analyses using the full cohort. Multivariable logistic regression analyses were performed to assess the independent association after adjusted metformin prescription after hospital admission and adjusted for propensity score. Additionally, A previous study excludes patients with myocardial infarction during the previous month, who were contraindicated to metformin[18]. We exclude patients admitted to CCU for sensitivity analyses. 


\section{Results}

\section{Population}

We identified 4,328 individuals with type 2 diabetes who underwent AKI according to the KDIGO definition. Of these patients, $998(23 \%)$ had preadmission metformin usage. Figure 1 presented the flow chart of the study patients. After propensity score matching, patient characteristics were balanced across groups (Table 1). The extent of missing values was presented in eTables 1 in the Supplement.

\section{Baseline characteristics}

The demographic characteristics of all participants were presented in eTable 1. Among the 982 pairs matched patients, the mean age was $67.0 \pm 12.6,797(40.6 \%)$ were female, 1376 (70.1\%) were white individuals, and 588 (29.9\%) were non-white individuals. The number of patients in each AKI stage within 7 days was 817(stage 1), 774 (stage 2), 373 (stage 3), respectively (Table 1,eTable 2).

On day 30 , the mortality was $15.7 \%(523 / 3,330)$ and $9.0 \%$ (90/998) in no preadmission metformin usage and preadmission metformin usage group, respectively (Table 2). The IPW demonstrated a significant beneficial effect of preadmission metformin usage in terms of 30-day mortality. The HR was $0.63(95 \%$ $\mathrm{Cl}, 0.50-0.80, \mathrm{P}<0.0001)$. The propensity score-matched mortality rates for the no preadmission metformin usage and preadmission metformin usage groups were $14.8 \%$ vs. $9 \%$. In univariable Cox proportional hazards regression, The HR was 0.59 (95\% Cl, 0.45-0.77, $\mathrm{P}<0.0001)$. Kaplan-Meier curve showed patients with preadmission metformin usage had lower mortality by day 30 (Log-rank test: $p<0.0001$, Figure 2).

\section{Secondary outcomes studies with propensity score matching}

We evaluated several secondary outcomes to investigate potential factors that might account for the beneficial effects of preadmission metformin usage after PSM. First, NLR had a numerical decrease among patients with preadmission metformin usage compared with those without preadmission metformin usage in admission[7.4 (4.1-13.0) VS 8.1 (4.5-13.8), $\mathrm{P}=0.08$ ]. Second, the metformin group had a significantly shorter duration of hospital stay [7.8 (5.1-12.8) VS 8.2 (5.5-13.9), $\mathrm{P}=0.022]$.

\section{Sensitive analysis}

In the full cohort $(\mathrm{N}=4,328)$, after adjusted for all covariates in table 1 , multivariable regression analysis similarly demonstrated that metformin was associated with a reduced hazard for 30 -day (HR=0.54; $95 \%$ $\mathrm{Cl}, 0.43-0.69, \mathrm{P}<0.0001)$ (Table 2). We further adjusted metformin prescription $(\mathrm{n}=780,18 \%)$ after hospital 
admission in multivariable regression analysis, the relationship remained stable $(\mathrm{HR}=0.66 ; 95 \% \mathrm{Cl}, 0.52-$ $0.84, \mathrm{P}=0.006)$. Additionally, we adjusted for propensity score, the $\mathrm{HR}$ was also similar $(\mathrm{HR}=0.63 ; 95 \% \mathrm{Cl}$, $0.50-0.79, \mathrm{P}<0.0001$ ) (Table 2).

In the full cohort, after excluding 705 patients admitted to $\mathrm{CCU}$, there were 3623 patients left, and the relationship between preadmission metformin usage and 30-day mortality stay reliable $(\mathrm{OR}=0.54$, $95 \% \mathrm{Cl}: 0.42-0.69, \mathrm{P}<0.001)$. In CCU admission patients, this relationship kept similar $(\mathrm{OR}=0.49$, $95 \% \mathrm{Cl}: 0.27-0.89, \mathrm{P}<0.020)$.

\section{Discussion}

In this retrospective, propensity score-matched cohort study, preadmission metformin usage provided to AKI patients with diabetes were associated with a lower risk-adjusted 30-day mortality, compared with no preadmission metformin usage. This association was reliable in additional models which control for the indicated bias.

In our study, 23.1\% $(998 / 4,328)$ AKI patients with type 2 diabetes received preadmission metformin exposure, which is lower compared with previous studies. Katherine et al. reported $59 \%$ of type 2 diabetes invidious used metformin before surgical procedure[19] and Samira et al. reported 40\% AKI patients type 2 diabetes used metformin[7]. The definition of metformin exposure may lead to this diversity. In MIMIC database metformin exposure was defined as the recent history of metformin use and in Katherine and Samira's cohort, metformin exposure was defined as prescribed metformin in the 180 days before surgical procedure or hospital admissions[7, 19].

In a multicenter cohort, CKD (eGFR 30-60 ml/min/1.732) patients treated with metformin may decrease mortality rates compared to those not on metformin[20]. A recent study also showed among patients with diabetes and reduced kidney function persisting with monotherapy, treatment with metformin, compared with a sulfonylurea, was associated with a lower risk of major adverse cardiovascular events[21]. These studies indicated metformin may benefit diabetes patients with kidney disease and U.S. Food and Drug Administration (FDA) has also revised their recommendation stating that it can be used in mild renal impairment and some with moderate renal impairment[22].

Similar to our findings, Bell et al. conducted an observational cohort study in Scotland $(n=4944)$ and described preadmission metformin was associated with a lower rate of 28-day mortality (HR 0.82;95\% $\mathrm{Cl}, 0.70-0.95, \mathrm{P}=0.01$ )[7]. Compared with our study, Bell et al. lacked data of critical care patients. In our cohort, we focused only on AKI patients in ICU and considered some critical risk factors such as SAPS II score, vasopressor usage, and ventilator usage. Thus, our study extended these findings by demonstrating that preadmission metformin usage was also associated with a lower risk of all-cause motility in AKI patients from ICU.

Alike findings in previous research, metformin was associated with lower NLR, which is a marker of systemic inflammation[23]. In other cohorts, a lower level of NLR is associated with a decreased risk of 
mortality in AKI patients[24, 25]. However, it remains unclear the potential mechanism of preadmission metformin usage associated with lower mortality in AKI patients with diabetes. Metformin could improve autophagy and mitochondrial function in diabetes[26]and decrease inflammation by down-regulate proinflammatory cytokines, such as IL-6 and TNF-a[27, 28]. Besides, metformin may have a potential role in antimicrobial therapy. Laboratory tests showed the effectiveness of metformin on multiple pathogens, including Trichinella spiralis, Staphylococcus aureus, Pseudomonas aeruginosa, hepatitis B virus, hepatitis $C$ virus, and human immunodeficiency virus[29]. The antimicrobial effect may be involved in the beneficial impact of sepsis. Furthermore, some studies show metformin could affect gut microbiota [30]and may benefit the AKI patients.

Several limitations are noteworthy. First, the potential for residual confoundings may exist, as with all retrospective analyses. We adjusted as many possible confoundings as we can and did a good balance in the PSM cohorts in this study. Second, as the study population contains only AKI patients with type 2 diabetes, it may not be generalizable to AKI patients with type 1 diabetes. Third, we were unable to exclude patients with myocardial infarction during the previous month, who were contraindicated to metformin[18]. We exclude patients admitted to CCU for instead. The result was still robust and reliable. Fourth, the record of metformin in "Medications on admission" in this study are likely to be more prone to unrecorded. The preadmission metformin usage in AKI patients with diabetes is lower than previously reported. However, it is noteworthy that the potential exposed misclassification resulting from such errors would bias toward the null, thus result in an underestimation of the association between preadmission metformin usage and 30-day mortality.

\section{Conclusions}

This cohort study suggested that preadmission metformin usage was associated with reduced riskadjusted mortality in AKI patients with type 2 diabetes. This association warrants further investigation.

\section{Abbreviations}

AKI: Acute kidney injury; Bpm: Beat per minute; CKD: Chronic kidney disease; ICU: Intensive care unit; SCr: Serum creatinine; ICD-9: International Classification of Disease, Ninth Revision; MAP: Mean arterial pressure; WBC: White blood cell; MIMIC: Medical Information Mart for Intensive Care; SD: Standard deviation; IQR: Interquartile range; SMD: Standardized mean difference; LOS: length of stay; CCU: Coronary care unit; CSRU: Cardiac surgery recovery unit; TSICU: Trauma and surgical intensive care unit; MICU: Medical intensive care unit; SICU: Surgical intensive care unit; NLR: Neutrophil-to-lymphocyte ratio; PSM: Propensity score matching; RRT: Renal replace treatment; SOFA: Sequential organ failure assessment; SAPS: Simplified acute physiology score;

\section{Declarations}

\section{Ethics approval and consent to participate}


The establishment of this database was approved by the Massachusetts Institute of Technology (Cambridge, MA) and Beth Israel Deaconess Medical Center (Boston, MA), and consent was obtained for the original data collection. Therefore, the ethical approval statement and the need for informed consent were waived for this manuscript.

\section{Consent for publication}

Not applicable

\section{Availability of data and materials}

Data in the article can be obtained from MIMIC-III database (https://mimic.physionet.org/)

\section{Competing interests}

The authors declare that they have no competing interests.

\section{Funding}

There was no external for this work.

\section{Authors' contributions}

Qilin Yang conducted data analysis and wrote the manuscript. Jiezhao Zheng conducted data analysis. Deliang Wen conducted data collection and data analysis. Xiaohua Chen conducted the data collection. Weiyan Chen conducted data collection and data interpretation. Weixiao chen drew the figure. Xuming Xiong designed the study and reviewed the manuscript. Zhenhui Zhang designed the study and reviewed the manuscript.

\section{Acknowledgments}

We thank Dr. Liu jie (People's Liberation Army of China General Hospital, Beijing, China) and Dr. Pan jiangang (The Second Affiliated Hospital of Guangzhou Medical University, Guangzhou, Guangdong, China) for helping in this revision.

\section{References}


1. Singbartl K, Kellum JA: AKI in the ICU: definition, epidemiology, risk stratification, and outcomes. KIDNEY INT 2012, 81(9):819-825.

2. Moore PK, Hsu RK, Liu KD: Management of Acute Kidney Injury: Core Curriculum 2018. AM J KIDNEY DIS 2018, 72(1):136-148.

3. Inzucchi SE, Bergenstal RM, Buse JB, Diamant M, Ferrannini E, Nauck M, Peters AL, Tsapas A, Wender R, Matthews DR: Management of Hyperglycemia in Type 2 Diabetes, 2015: A PatientCentered Approach: Update to a Position Statement of the American Diabetes Association and the European Association for the Study of Diabetes. DIABETES CARE 2015, 38(1):140-149.

4. Cameron AR, Morrison VL, Levin D, Mohan M, Forteath C, Beall C, Mcneilly AD, Balfour DJK, Savinko T, Wong AKF: Anti-Inflammatory Effects of Metformin Irrespective of Diabetes StatusNovelty and Significance. CIRC RES 2016, 119(5):652-665.

5. Crowley MJ, Diamantidis CJ, McDuffie JR, Cameron CB, Stanifer JW, Mock CK, Wang X, Tang S, Nagi A, Kosinski AS et al: Clinical Outcomes of Metformin Use in Populations With Chronic Kidney Disease, Congestive Heart Failure, or Chronic Liver Disease: A Systematic Review. ANN INTERN MED 2017, 166(3):191-200.

6. Kwon S, Kim YC, Park JY, Lee J, An JN, Kim CT, Oh S, Park S, Kim DK, Oh YK et al: The Long-term Effects of Metformin on Patients With Type 2 Diabetic Kidney Disease. DIABETES CARE 2020, 43(5):948-955.

7. Bell S, Farran B, McGurnaghan S, McCrimmon RJ, Leese GP, Petrie JR, McKeigue P, Sattar N, Wild S, McKnight $\mathrm{J}$ et al: Risk of acute kidney injury and survival in patients treated with Metformin: an observational cohort study. BMC NEPHROL 2017, 18(1):163.

8. Hoste EAJ, Bagshaw SM, Bellomo R, Cely CM, Colman R, Cruz DN, Edipidis K, Forni LG, Gomersall CD, Govil $\mathrm{D}$ et al: Epidemiology of acute kidney injury in critically ill patients: the multinational AKI-EPI study. INTENS CARE MED 2015, 41(8):1411-1423.

9. Johnson AEW, Pollard TJ, Lu S, Lehman LWH, Mark RG: MIMIC-III, a freely accessible critical care database. SCI DATA 2016, 3:160035.

10. Elm EV, Altman DG, Egger M, Pocock SJ, Gøtzsche PC, Vandenbroucke JP: Strengthening the reporting of observational studies in epidemiology (STROBE) statement: guidelines for reporting observational studies. BMJ 2007, 335(7624):806-808.

11. Levin A, Stevens PE, Bilous RW: Kidney disease: Improving global outcomes (KDIGO) CKD work group. KDIGO 2012 clinical practice guideline for the evaluation and management of chronic kidney disease. KIDNEY INT SUPPL 2013, 3(1):1.

12. Zhao G, Xu C, Ying J, Lü W, Hong G, Li M, Wu B, Yao Y, Lu Z: Association between furosemide administration and outcomes in critically ill patients with acute kidney injury. CRIT CARE 2020, 24(1).

13. Huber M, Ozrazgat-Baslanti T, Thottakkara P, Scali S, Hobson C: Cardiovascular-Specific Mortality and Kidney Disease in Patients Undergoing Vascular Surgery. JAMA SURG 2015, 151(5):1-10.

14. Angeli P, Ginès P, Wong F, Bernardi M, Boyer TD, Gerbes A, Moreau R, Jalan R, Sarin SK, Piano S: Diagnosis and management of acute kidney injury in patients with cirrhosis: Revised consensus 
recommendations of the International Club of Ascites. J HEPATOL 2015, 62(4):968-974.

15. Shrank WH, Patrick AR, Brookhart MA: Healthy User and Related Biases in Observational Studies of Preventive Interventions: A Primer for Physicians. J GEN INTERN MED 2011, 26(5):546-550.

16. Shen Y, Zhang W, Shen Y: Early diuretic use and mortality in critically ill patients with vasopressor support: a propensity score-matching analysis. CRIT CARE 2019, 23(1).

17. Feng M, Mcsparron JI, Trung KD, Stone DJ, Roberts DH, Schwartzstein RM, Antoine VB, Anthony CL: Transthoracic echocardiography and mortality in sepsis: analysis of the MIMIC-III database. INTENS CARE MED 2018.

18. Jochmans S, Alphonsine J, Chelly J, Vong LVP, Sy O, Rolin N, Ellrodt O, Monchi M, Vinsonneau C: Does metformin exposure before ICU stay have any impact on patients' outcome? A retrospective cohort study of diabetic patients. ANN INTENSIVE CARE 2017, 7(1):116.

19. Reitz KM, Marroquin OC, Zenati MS, Kennedy J, Korytkowski M, Tzeng E, Koscum S, Newhouse D, Garcia RM, Vates J et al: Association Between Preoperative Metformin Exposure and Postoperative Outcomes in Adults With Type 2 Diabetes. JAMA SURG 2020:e200416.

20. Roussel R: Metformin Use and Mortality Among Patients With Diabetes and Atherothrombosis<alttitle>Metformin Use With Diabetes and Atherothrombosis</alt-title>. Archives of Internal Medicine 2010, 170(21):1892.

21. Roumie CL, Chipman J, Min JY, Hackstadt AJ, Hung AM, Greevy RAJ, Grijalva CG, Elasy T, Griffin MR: Association of Treatment With Metformin vs Sulfonylurea With Major Adverse Cardiovascular Events Among Patients With Diabetes and Reduced Kidney Function. JAMA 2019, 322(12):1-11.

22. FDA Drug Safety Communication: FDA revises warnings regarding use of the diabetes medicine metformin in certain patients with reduced kidney function [http://www.fda.gov/downloads/Drugs/DrugSafety/UCM494140.pdf]. Accessed 07/20/2020.

23. Cameron AR, Morrison VL, Levin D, Mohan M, Forteath C, Beall C, McNeilly AD, Balfour DJK, Savinko T, Wong AKF et al: Anti-Inflammatory Effects of Metformin Irrespective of Diabetes Status. CIRC RES 2016, 119(5):652-665.

24. Fan LL, Wang YJ, Nan CJ, Chen YH, Su HX: Neutrophil-lymphocyte ratio is associated with all-cause mortality among critically ill patients with acute kidney injury. Clinica chimica acta; international journal of clinical chemistry 2019, 490:207-213.

25. Kim HY, Kong Y, Park JH, Kim Y: Acute kidney injury after burn surgery: Preoperative neutrophil/lymphocyte ratio as a predictive factor. ACTA ANAESTH SCAND 2019, 63(2):240-247.

26. Bharath LP, Agrawal M, McCambridge G, Nicholas DA, Hasturk H, Liu J, Jiang K, Liu R, Guo Z, Deeney $\mathrm{J}$ et al: Metformin Enhances Autophagy and Normalizes Mitochondrial Function to AlleviateAgingAssociated Inflammation. CELL METAB 2020.

27. Jing Y, Wu F, Li D, Yang L, Li Q, Li R: Metformin improves obesity-associated inflammation by altering macrophages polarization. MOL CELL ENDOCRINOL 2018, 461:256-264.

28. Cameron AR, Morrison VL, Levin D, Mohan M, Forteath C, Beall C, McNeilly AD, Balfour DJK, Savinko T, Wong AKF et al: Anti-Inflammatory Effects of Metformin Irrespective of Diabetes Status. CIRC RES 
2016, 119(5):652-665.

29. Malik F, Mehdi SF, Ali H, Patel P, Basharat A, Kumar A, Ashok F, Stein J, Brima W, Malhotra P et al: Is metformin poised for a second career as an antimicrobial? Diabetes/Metabolism Research and Reviews 2018, 34(4):e2975.

30. Lee H, Lee Y, Kim J, An J, Lee S, Kong H, Song Y, Lee C, Kim K: Modulation of the gut microbiota by metformin improves metabolic profiles in aged obese mice. Gut microbes 2018, 9(2):155-165.

\section{Tables}

Table 1. Baseline characteristics of participants 
Unmatched Patients

No. $(\%)$

Patient

characteristic

No metformin

$(\mathrm{n}=3330 \rrbracket$

$68.5 \pm 13.0$

Age(years)

Female, sex, no. $\quad 1898(57.0)$

(\%)

Ethnicity, non-

white, no.(\%)

Marital status, no.(\%)

Married

Other

1631 (49.0)

$1699(51.0)$

Insurance, no.(\%)

Medicaid

Private

Other

Service unit, no.(\%)

CCU

CSRU

MICU

SICU

TSICU

Heart rate (bpm)

MAP $(\mathrm{mmHg})$

Respiratory rate $\quad 18.9 \pm 4.0$

(bpmヌ

$\mathrm{SPO}_{2}(\%)$

Glucose $(\mathrm{mg} / \mathrm{dL}) \quad 165.6 \pm 53.6$

WBC $\left(\times 10^{9}\right.$

\section{$1147(34.4)$}

2442 (73.3)

$810(24.3)$

$78(2.3)$

$562(16.9)$

$770(23.1)$

$1210(36.3)$

479 (14.4)

$309(9.3)$

$85.3 \pm 15.3$

$76.9 \pm 10.6$

$97.3 \pm 2.3$

$14.6 \pm 8.7$
$292(29.3)$

$0.11 \quad 301(30.7)$

0.09

$532(53.3)$

$466(46.7)$
$97.2 \pm 1.9$

$164.4 \pm$

51.0

$642(64.3)$

0.16

$0.16280(28.5)$

$0.01 \quad 35(3.6)$

$143(14.3)$

$0.06 \quad 164(16.7)$

$0.14 \quad 256(26.1)$

$326(33.2)$

$142(14.2) \quad<0.01 \quad 137(14)$

$<0.01 \quad 99(10.1)$

$19.1 \pm 3.7 \quad 0.06 \quad 19.2 \pm 4.3$

$0.04 \quad 97.3 \pm 2.3$

$0.02 \quad 164.9 \pm 52.6$

$15.3 \pm 18.6$

$86.5 \pm 14.6 \quad 0.08 \quad 85.8 \pm 15.3 \quad 86.5 \pm 14.7 \quad 0.04$

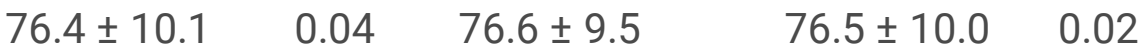

Metformin SMD $(n=982 \rrbracket$

$66.7 \pm 11.8 \quad 0.05$ $592(60.3) \quad 0.04$ $287(29.2) \quad 0.03$ 0.02

$525(53.5)$

$457(46.5)$

$633(64.5) \quad 0.07$
$325(33.1) \quad 0.10$

$24(2.4)$

0.07

$19.1 \pm 3.7$

0.01

$97.2 \pm 1.9 \quad 0.04$

$164.3 \pm$

0.01 50.5

$14.8 \pm 9.0 \quad 0.06$ 


\begin{tabular}{|c|c|c|c|c|c|c|}
\hline $\begin{array}{l}\text { Creatinine } \\
(\mathrm{mg} / \mathrm{dL})\end{array}$ & $1.5 \pm 1.0$ & $1.5 \pm 1.5$ & 0.02 & $1.5 \pm 1.3$ & $1.4 \pm 1.3$ & 0.06 \\
\hline $\begin{array}{l}\text { Hemoglobin } \\
(\mathrm{g} / \mathrm{L})\end{array}$ & $9.9 \pm 2.1$ & $9.7 \pm 2.2$ & 0.07 & $9.8 \pm 2.1$ & $9.7 \pm 2.2$ & 0.04 \\
\hline Platelet $\left(\times 10^{12}\right)$ & $195.3 \pm 101.2$ & $\begin{array}{l}199.0 \pm \\
96.8\end{array}$ & 0.04 & $193.6 \pm 98.3$ & $\begin{array}{l}198.7 \pm \\
96.4\end{array}$ & 0.05 \\
\hline SAPS II score & $38.6 \pm 13.8$ & $37.0 \pm 13.0$ & 0.12 & $38.1 \pm 13.9$ & $37.1 \pm 13.0$ & 0.08 \\
\hline $\begin{array}{l}\text { Ventilator use, n } \\
(\%)\end{array}$ & 1859 (55.8) & $604(60.5)$ & 0.10 & 570 (58) & 599 (61) & 0.06 \\
\hline $\begin{array}{l}\text { Vasopressor use, } \\
\text { n (\%) }\end{array}$ & $1474(44.3)$ & $470(47.1)$ & 0.06 & $464(47.3)$ & $465(47.4)$ & $\begin{array}{l}\square \\
0.01\end{array}$ \\
\hline Infection, n (\%) & $1623(48.7)$ & $427(42.8)$ & 0.12 & $455(46.3)$ & 421 (42.9) & 0.07 \\
\hline RRT, n (\%) & $116(3.5)$ & $31(3.1)$ & 0.02 & $27(2.7)$ & $30(3.1)$ & 0.02 \\
\hline \multicolumn{7}{|l|}{ AKI stage } \\
\hline Stage 1 & 1317 (39.5) & 423 (42.4) & 0.05 & $401(40.8)$ & 416 (42.4) & 0.03 \\
\hline Stage 2 & 1319 (39.6) & 389 (39.0) & 0.04 & $388(39.5)$ & 386 (39.3) & 0.01 \\
\hline Stage 3 & $694(20.8)$ & $186(18.6)$ & 0.05 & 193 (19.7) & $180(18.3)$ & 0.03 \\
\hline \multicolumn{7}{|c|}{ Comorbidity disease (\%) } \\
\hline CHF & $563(16.9)$ & 187 (18.7) & 0.05 & $164(16.7)$ & $184(18.7)$ & 0.05 \\
\hline Liver disease & $257(7.7)$ & $66(6.6)$ & 0.04 & $62(6.3)$ & $66(6.7)$ & 0.02 \\
\hline CAD & $1352(40.6)$ & $447(44.8)$ & 0.08 & $444(45.2)$ & $441(44.9)$ & 0.01 \\
\hline Stroke & $358(10.8)$ & $104(10.4)$ & 0.01 & $121(12.3)$ & $101(10.3)$ & 0.06 \\
\hline Malignancy & 496 (14.9) & $163(16.3)$ & 0.04 & 155 (15.8) & $162(16.5)$ & 0.02 \\
\hline $\begin{array}{l}\text { Respiratory } \\
\text { failure }\end{array}$ & 1049 (31.5) & $297(29.8)$ & 0.04 & $287(29.2)$ & 289 (29.4) & $\begin{array}{l}\square \\
0.01\end{array}$ \\
\hline
\end{tabular}

Bpm: beat per minute, CAD: coronary heart disease, CCU: coronary care unit, CHF:

Congestive heart failure, CSRU: cardiac surgery recovery unit, MICU: medical intensive care unit, MAP: mean arterial pressure, RRT: renal replace treatment, SICU: surgical intensive care unit, SAPS: simplified acute physiology score, TSICU: trauma and surgical intensive care unit, WBC: white blood count. 
Table 2. Associations between metformin use and the outcome in the crude analysis, multivariable analysis, and propensity-score analyses.

\begin{tabular}{|lll|}
\hline Analysis & 30-day mortality (\%) & P-value \\
\hline No. of events/no. of patients at risk (\%) & & \\
\hline No metformin use & $523 / 3,330(15.7)$ & \\
\hline Metformin use & $90 / 998(9.0)$ & \\
\hline Crude analysis - hazard ratio $(95 \% \mathrm{Cl})$ & $0.57(0.44,0.69)$ & $<0.0001$ \\
\hline Multivariable analysis - hazard ratio $(95 \% \mathrm{Cl}) *$ & $0.54(0.43,0.69)$ & $<0.0001$ \\
\hline With inverse probability weighting $\dagger$ & $0.63(0.50,0.80)$ & $<0.0001$ \\
\hline With matching $\ddagger$ & $0.59(0.45,0.77)$ & $<0.0001$ \\
\hline Adjusted for propensity score $\S$ & $0.63(0.50,0.79)$ & $<0.0001$ \\
\hline
\end{tabular}

* Shown is the hazard ratio from the multivariable Cox proportional-hazards model, with adjusted for all covariates in table 1.

† Shown is the primary analysis with a hazard ratio from the multivariable Cox proportional-hazards model with the same strata and covariates with inverse probability weighting according to the propensity score.

¥ Shown is the hazard ratio from a multivariable Cox proportional-hazards model with the same strata and covariates with matching according to the propensity score. The analysis included 982 patients (982 who received preadmission metformin prescription and 982 who did not).

$\S$ Shown is the hazard ratio from a multivariable Cox proportional-hazards model with the same strata and covariates, with additional adjustment for the propensity score.

\section{Figures}




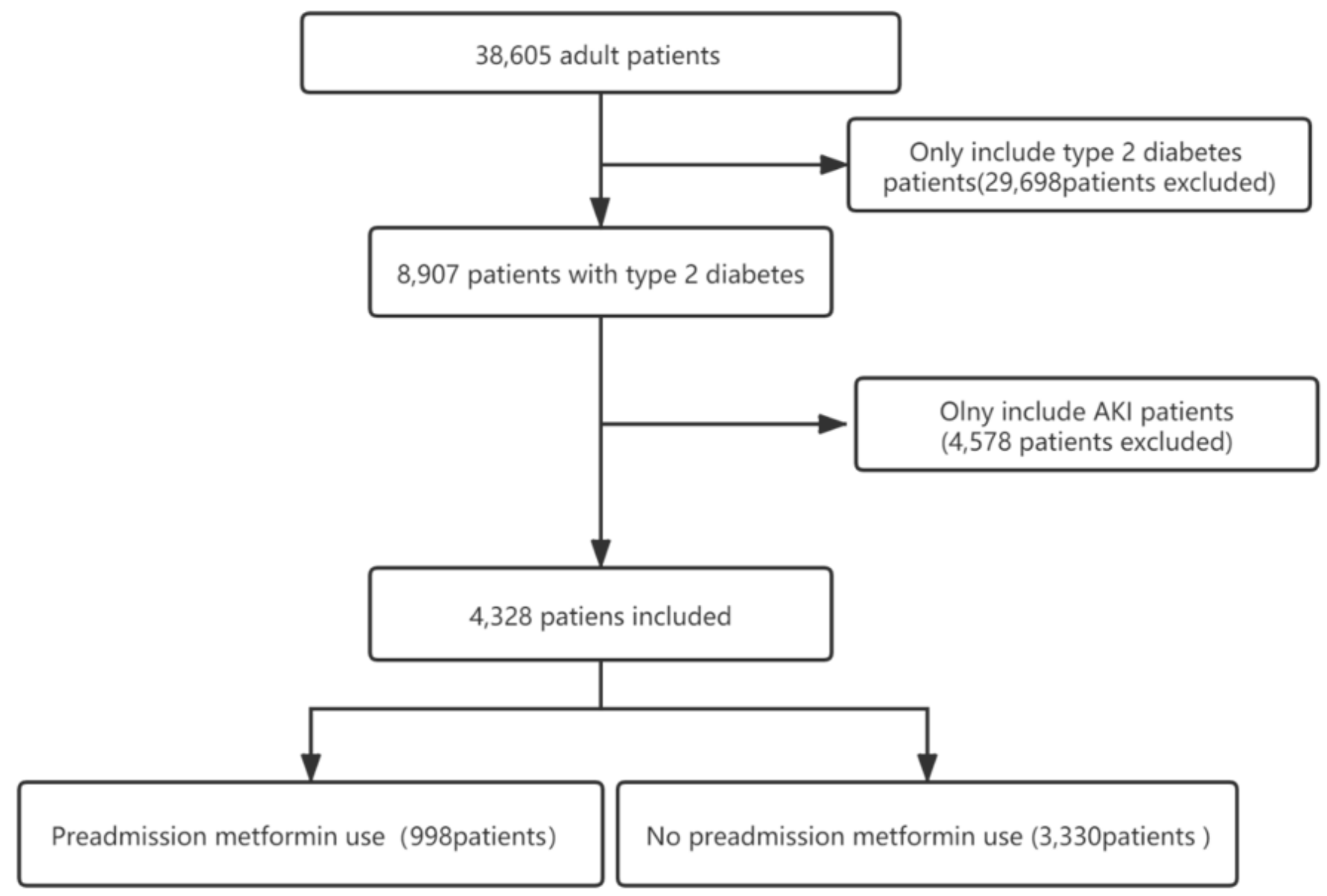

\section{Figure 1}

The flow chart of the study 


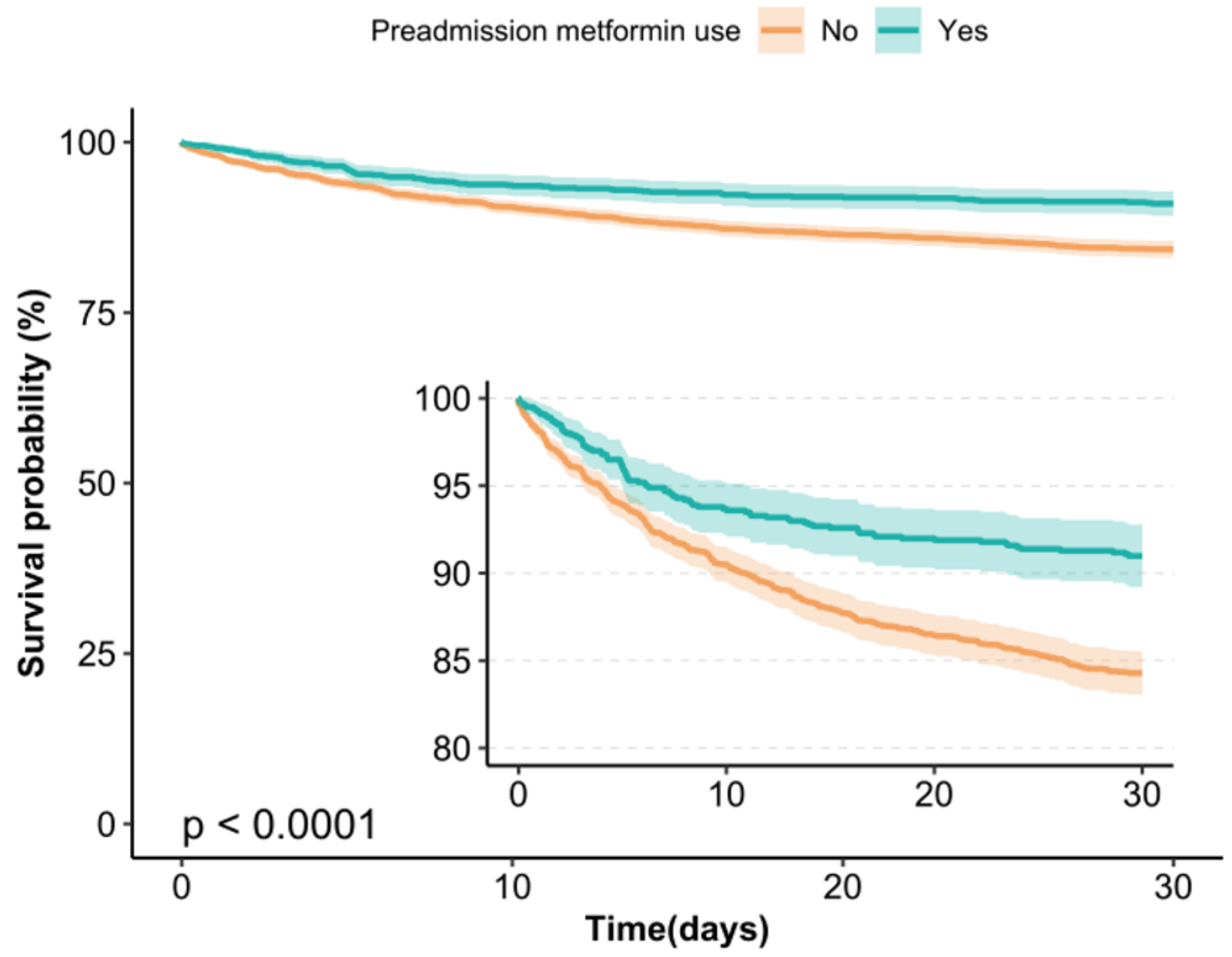

Number at risk

\begin{tabular}{|c|c|c|}
\hline No 3330 & 3014 & 2879 \\
\hline 998 & 935 & 918 \\
\hline
\end{tabular}

Figure 2

Kaplan-Meier Survival Curves for day 30 of AKI patients with type 2 diabetes

\section{Supplementary Files}

This is a list of supplementary files associated with this preprint. Click to download.

- etable.docx 